\title{
XIX
}

\section{SAÚDE MENTAL E O TRABALHO INTERSETORIAL: ESTRATÉGIAS E DESAFIOS PARA A ATENÇÃO PSICOSSOCIAL EM TEMPOS DE ISOLAMENTO SOCIAL*}

Anália da Silva Barbosa

Magda Costa Barreto

\section{Introdução}

O presente capítulo tem como objetivo, a partir das experiências vivenciadas por duas assistentes sociais e gestoras de diferentes Centros de Atenção Psicossocial (CAPS) e das discussões teóricas que norteiam o cotidiano profissional, apresentar e discutir as estratégias e os desafios colocados para desenvolvimento do trabalho intersetorial no campo da Atenção Psicossocial tendo como pano de fundo o período de isolamento social ao longo da Pandemia do COVID 19.

Nesse caminho, temos como dado que a COVID-19 foi declarada como uma emergência de saúde pública, em 30 de janeiro de 2020, pela Organização Mundial da Saúde (OMS). Desde então, em âmbito global, os países, de diferentes maneiras, empreendem esforços para organizar seus serviços de saúde, e nessa conjuntura, ressalta-se a particularidade de grupos socialmente vulneráveis que estão mais expostos ao risco por encontrarem-se em condições mais precárias de vida.

Contudo destaca-se que a Pandemia do COVID-19 encontra o Brasil em um cenário que desde 2014 tem sofrido ataques às políticas sociais, principalmente após 2016, com a adesão das políticas ultraneoliberais e que desmontam os direitos conquistados fragilizando os sistemas públicos que compõem a proteção social.

Sendo assim, diante desse cenário, pensar intersetorialidade e o campo da atenção psicossocial demanda refletir acerca do pro- 
cesso de trabalho pautado em uma concepção ampliada de saúde, implicada numa relação que envolve o contexto econômico, social e cultural do país; logo, abrangendo situações de moradia, saneamento, renda, alimentação, educação, acesso ao lazer e bens (SCHEFFER; SILVA, 2014).

\section{Contextualizando a reforma psiquiátrica e a rede de aten- ção psicossocial}

Para compreender a Rede de Atenção à Saúde Mental Brasileira faz-se necessário saber que a mesma é parte integrante do Sistema Único de Saúde (SUS) o que inclui ações e serviços públicos de saúde. Logo, temos como premissa na Política Nacional de Saúde Mental (PNSM) a busca pela consolidação de um modelo de atenção à saúde mental aberto e de base comunitária, que possui uma rede de serviços e equipamentos variados tais como as diferentes modalidades de Centros de Atenção Psicossocial (CAPS), os Serviços Residenciais Terapêuticos (SRT), os Centros de Convivência e Cultura e os leitos de atenção integral em Hospitais Gerais, bem como, os equipamentos da atenção básica de saúde.

Partindo dessa compreensão, para constituir essa rede, todos os recursos afetivos (relações pessoais, familiares, amigos), sociais (moradia, trabalho, escola, esporte), econômicos (dinheiro, previdência), culturais e de lazer são convocados para potencializar as equipes de saúde nos esforços de cuidado e reabilitação psicossocial (MINISTÉRIO DA SAÚDE, 2004)

Logo, a Reforma Psiquiátrica Brasileira afirma que as práticas de saúde mental produzem um cuidado que extrapola o campo biomédico, marcando-as por uma condução teórico-ético-metodológica, que coaduna na direção a uma proposta de mudança societária através de ações comunitárias e territoriais voltados para o direito à moradia, ao trabalho e ao lazer dos usuários, tendo o território como espaço que viabiliza conexões de produção de vida dos sujeitos.

Partindo dessa lógica, nesse percurso, em 2010 temos a IV Conferência Nacional de Saúde Mental, a primeira convocada como intersetorial, em que Amarante e Nunes (2018), apontam que esta 
aconteceu após intensa pressão dos movimentos sociais que realizaram várias manifestações e a partir da organização da histórica Marcha dos Usuários, em Brasília. Porém destacam que apesar de nomeada como intersetorial, teve uma participação incipiente de outros setores de movimentos sociais ligados ao trabalho, direitos humanos, cultura, educação, direito à terra e assim por diante.

Em 2011, o Conselho Nacional de Saúde, através da resolução no. 488 determina o aumento de recursos e ampliação da Rede de Atenção Psicossocial (RAPS) para que se cumpra à atuação e articulação intersetorial (BRASIL, 2011). Com esta perspectiva, a portaria $\mathrm{n}$ ㅇ 3.088 instituirá a RAPS para pessoas com sofrimento ou transtorno mental e com necessidades decorrentes do uso de crack, álcool e outras drogas, no âmbito do SUS (BRASIL, 2011).

Com dezessete artigos, a portaria apresenta as diretrizes, objetivos gerais e específicos, componentes da RAPS (atenção básica, atenção especializada, urgência e emergência, atenção residencial e rede hospitalar) e seus pontos de atenção. A portaria traz a importância da criação de diferentes dispositivos territoriais e a necessidade da integração entre eles, especialmente com a Atenção Básica. "Organização dos serviços em rede de atenção à saúde regionalizada, com estabelecimento de ações intersetoriais para garantir a integralidade do cuidado" (BRASIL, 2011, art. 2 inciso X).

Contudo, ao mesmo tempo em que traz a diretriz e determina a criação de dispositivos importantes, na perspectiva territorial e intersetorial, a RAPS permite a inclusão das comunidades terapêuticas (CT), as quais vêm a fazer parte do componente de atenção residencial de caráter transitório. A inclusão das CT na RAPS reforça a possibilidade de atuação da esfera privada no SUS que recebem financiamento para realizarem um "cuidado" que é contrário aos princípios da Reforma Psiquiátrica.

Acrescido a isso, temos as alterações nas leis e documentos ministeriais no período de 2010-2019, com o Decreto 7.179 de 2010, que institui o Plano Integrado de Enfrentamento ao Crack e Outras Drogas, e, em 2017, diante de uma conjuntura política profundamente adversa, a Portaria 3.588, e, reafirmados, em 2019, pela Nota Técnica 11/2019 que trouxe alterações na PNSM, atacando os princípios da Reforma Psiquiátrica Brasileira e os direitos garantidos, uma vez 
que fortalece a lógica privatizante do mercado (GUIMARÃES; ROSA, 2019).

Duarte (2018) apresenta as contradições desde o processo de construção da RAPS, e as ostensivas mudanças diante da nova política de saúde mental (BRASIL, 2017) e da nova política de drogas, através da Resolução 01/2018 (Brasil, 2018) que, segundo o autor, contribuem com o proibicionismo e manicomialização.

Assim selando o golpe na área, com uma lógica médico manicomial, a "nova" RAPS tem os seguintes dispositivos: Equipe multiprofissional de atenção especializada em saúde mental/unidades ambulatoriais especializadas, unidade de referência especializada em hospital geral, hospital psiquiátrico especializado, hospital dia e CAPSad IV/R, que funcionará $24 \mathrm{~h}$, com equipes multiprofissionais e que serão localizados em regiões metropolitanas, acima de 500 mil habitantes, próximos às "cracolândias" (DUARTE, 2018, p. 235).

Dentre as controvérsias da Portaria, destaca-se a inclusão dos hospitais psiquiátricos na RAPS e o aumento do financiamento do valor da sua diária, concedendo reajuste acima de $60 \%$ - enquanto não há aumento do repasse para os CAPS desde 2011, conforme Guimarães e Rosa (2019).

Logo, no contexto atual, temos outras formas manicomiais e asilares, retrocessos no campo legislativo, na implantação e forte presença do discurso conservador. Sendo assim, identificamos que no início do Movimento de Reforma Psiquiátrica (MRP) vivíamos em outro contexto social, hoje, temos novos direitos e novos sujeitos (AMARANTE, 1995).

Acrescido a isso, ocorreu uma reestruturação e mudanças na sociedade, no modo de viver, de maneira mais veemente situações desemprego em massa, pobreza, além dos congelamentos dos gastos com as políticas sociais. Observa-se o incentivo em massa ao consumo excessivo em concomitância com precárias condições de vida, sendo assim, diante de uma sociedade adoecida e massacrada pelas suas contradições, as necessidades mudam!

Para Duarte e Coe (2017), a atenção psicossocial é um campo que congrega um conjunto de práticas substitutivas ao modelo asilar, através de liberdade para experimentação e participação popular, 
que possibilita encontro com situações que estão se processando e abertura para outras que surgirão.

Diante disso, a atenção psicossocial se configura como um campo que tem por objetivo romper com as lógicas manicomiais, que se reconfiguram de acordo como o contexto político e social. Se, inicialmente, a principal preocupação era o fechamento dos grandes hospitais manicomiais carregados pelo saber da Psiquiatria, percorremos um período de esforços para o avanço do aparato legislativo legal, discussões mais ampliadas sobre as práticas e saberes que corroboraram para o trabalho comunitário em rede.

O cuidado demandado à Saúde Mental e a consolidação do fechamento dos manicômios e desinstitucionalização dos chamados usuários crônicos e dos sujeitos em novos processos de adoecimento, enfrenta o desafio de construção do cuidado integral, que é atravancado pelo sucateamento das políticas sociais, pela ideologia do especialismo que contribui para ênfase nos serviços especializados.

A compreensão de Basaglia (2005), Rotelli (2008), Amarante (2007), Nicácio (2005), Lancetti (2009) entre outros caminhos na direção de uma prática comunitária em saúde mental promovendo para além de uma intervenção de uma clínica restrita, logo, entendendo que a importância da restituição da contratualidade social entre usuário, familiares, técnicos e comunidade é o que produz sujeitos conscientes, ativos e críticos.

Então, nós temos muitas coisas a aprender: como colocar de pé as condições que possam reproduzir uma vida [...] aprender que a liberdade é terapêutica. Mas a liberdade só é terapêutica se não significar deixar as pessoas sós. A liberdade é um fato coletivo [...]. Devemos ser muito mais incisivos na transformação do mundo (ROTELLI, 2008, p. 41 e 46).

Dito isto, temos a potência dos Centros de Atenção Psicossocial, que apesar de todos os atravessamentos impostos a sua implantação de forma ampliada, vem contribuindo significativamente para o cuidado no território. Logo, para os CAPS, o território assume um significado inovador, diferente de um mero espaço geográfico, sendo produtor de contradições, lutas, produções sociais e ideológicas da vida concreta e simbólica em um processo histórico (YASUI, 2010). 
Nesse contexto brasileiro, a Reforma Psiquiátrica e a possibilidade de ampliação do trabalho do CAPS chega ao século XXI com muitos desafios, mas com importantes avanços, no sentido de ampliação, mesmo que insuficiente, dos dispositivos extra-hospitalares e concepção de cuidado ampliado em saúde mental, na lógica do cuidado integral, que para tanto, demanda uma articulação intra e intersetorial. Esse é um dos desafios que está posto! E para tanto é preciso reconhecer o que estamos entendendo por trabalho intersetorial, quem são esses outros setores e atores?

\section{Intersetorialidade no campo saúde mental}

Tendo em vista, os elementos supracitados em relação ao campo da Atenção Psicossocial, os serviços territoriais/antimanicomiais da RAPS, em particular os CAPS, é possível reconhecer a intersetorialidade como imprescindível para atuação neste campo. Mas afinal, o que estamos entendendo por intersetorialidade? E por que essa estratégia é imprescindível para o cuidado no campo da Atenção Psicossocial?

A intersetorialidade aparece no Brasil, a partir da Constituição Federal de 88, em que, no artigo 14, ressalta a importância da articulação das políticas de Saúde, Assistência Social e Previdência Social que compõe o tripé da seguridade social. E aos poucos vai sendo reconhecida como uma diretriz para várias políticas, na medida em que possibilita uma visão mais integrada dos problemas sociais (PEREIRA, 2020).

Partindo dessa trajetória a intersetorialidade passa a ser reconhecida como estratégia de gestão pública que tem como objetivo a articulação e complementarização entre os setores, que pode contribuir para uma visão mais ampliada das expressões da questão social, que estão relacionadas como as questões estruturais da sociedade capitalista. Desta forma, a intersetorialidade busca ações integradas de diferentes setores e segmentos que deve fazer o esforço para atender as demandas sociais da população em sua integralidade.

Wanderley, Martinelli e Paz (2020) destacam outro ponto em relação a intersetorialidade, a importância de compreendê-la além da 
iniciativa individual de profissionais e equipes, pois a mesma requer decisões institucionais e planejamento, para assim, conseguir superar a lógica da setorização e fragmentação.

Falar da intersetorialidade na Saúde Mental implica reconhecer que é uma questão que vai além do tratamento da doença, envolve fatores sociais complexos exigindo que tenhamos clareza que na medida em que as complexidades avançam as diretrizes da intersetorialidade começam a ser utilizadas, e consequentemente aumenta-se a capacidade de intervenção acerca de uma mesma situação, de modo a reduzir as resistências instauradas (BIDARRA, 2009, p.490).

Soma-se a esse cenário o desmonte dos direitos e das políticas sociais em curso no Brasil desde o golpe parlamentar de 2016, e aprofundadas com a implementação da agenda de reformas do governo do presidente Jair Bolsonaro, as quais atacam a cidadania e a democracia, destruindo os avanços alcançados nos últimos 30 anos e buscando a implantação de Estado mínimo liberal (WANDERLEY; MARTINELLI; PAZ, 2020. p. 10).

Vamos à segunda questão!

Rotelli (1990), em a Instituição Inventada, faz uma crítica à Psiquiatria cujo foco é o objeto doença, apartada do corpo social. Para ele, o objeto deve ser analisado incluindo a complexidade da sociedade, e assim, o objeto da saúde mental seria: a existência-sofrimento e sua relação com o corpo social.

Compactuamos com os apontamentos do autor, e acrescentamos que os usuários atendidos nos serviços da atenção psicossocial, não são sujeitos abstratos, eles têm cor, gênero, sexualidade, classe social, são sujeitos históricos e sociais. E diante disso, é importante reconhecer que a condição de vida que Ihes são impostas são também produtoras do adoecimento e fazem com que apresentem demandas concretas e complexas, que não podem ser reduzidas às questões somente biológicas e/ou biomédicas. Para tanto, é necessária uma análise social crítica da realidade, e uma atuação norteada pela clínica ampliada (DUARTE, 2011) que reconhece os diferentes saberes, a multiplicidade de serviços e recursos, para além da intervenção do setor saúde e das políticas sociais. 
Ao retratarmos o processo de Reforma Psiquiátrica e a implantação dos serviços substitutivos, é necessária a atenção ao encapsulamento, ou seja, Amarante (2007, p.86) recomenda que as equipes da atenção psicossocial "devem sair da sede do serviço e buscar na sociedade vínculos que complementem e ampliem os recursos existentes", uma vez que considera a intersetorialidade um conjunto de "estratégias que perpassam vários setores sociais, tanto do campo da saúde mental e saúde em geral, quanto das políticas públicas e da sociedade como o todo".

Portanto, consideramos a ação intersetorial como uma possibilidade de prática social, com viés democratizador que exige a participação dos movimentos sociais nos processos decisórios, insta paciência, tempo, flexibilidade, investimento nas políticas públicas, compartilhamento de saber e de poder, compromisso com a cidadania e promoção da condição humana.

É reconhecida a significativa importância dos CAPS, para o processo de fechamento dos manicômios e a mudança na lógica do trabalho na saúde mental, mas a centralidade nos CAPS, e sua lógica de funcionamento, podem trazer desafios para o trabalho intersetorial. Além da proposta de incorporação de outros serviços na rede RAPS (2011) como já discutido anteriormente, temos avanços importantes por meio de discussões relacionadas às questões societárias e macropolíticas (PASSOS, 2017), a discussão sobre, trabalho, gênero e classe (PEREIRA; PASSOS, 2017), racismo (PASSOS, 2018), diversidade sexual (DUARTE, 2017) e o campo da atenção psicossocial.

Elucidar essas discussões torna mais claro que é fundamental que no cotidiano dos serviços seja necessária uma atuação, que envolva as demais políticas sociais e seus atores, isso significa, segundo Silva e Magalhães Júnior (2008), que a construção de uma rede de saúde é produto de diversas políticas públicas e de condições historicamente acumuladas.

Destacamos também que cabe o reconhecimento das necessidades concretas dos usuários no cotidiano do serviço que demandam articulações intersetoriais de forma institucional para garantia do acesso a direitos diante das demais políticas sociais.

Vejamos alguns exemplos: no que se refere a política de Assistência Social a importância do acesso à documentação civil, o cadas- 
tramento para Bolsa Família, o Benefício de Prestação Continuada, assim como, o acolhimento institucional para população em situação de rua, abrigo protegido para mulheres que sofrem violência por parte de seus companheiros, dentre outras questões. Tudo isso sem excluir ações mais emergentes que envolvem as políticas de geração de renda e trabalho, como o cadastramento de currículo, com vias a vaga de trabalho, articulação com instituições para o trabalho protegido, capacitações e cursos profissionalizantes. Além do acesso ao lazer e cultura, a exposições, livros, teatro, artes, etc.

Wanderley, Martinelli e Paz, (2020) ampliam a discussão da intersetorialidade e vão ao encontro com o que acreditamos acerca da potência desse trabalho em rede, trazem que é nos territórios que intersetorialidade se concretiza. Dessa forma é preciso pensar também a intersetorialidade de forma ampla, e articulada com o trabalho em uma rede viva, e se é viva, precisa incluir as vivências e a realidade dos usuários, serviços e comunidades.

E assim, é necessário reconhecer, a importância de algumas vivências conforme relataremos. A importância do bar para um usuário atendido em um CAPS AD, sendo ele um senhor que vive sozinho, alcoolista crônico, uma vez que é nesse bar que ele é lembrado sobre os dias no CAPS e seu Projeto Terapêutico Singular (PTS), onde ele faz suas poucas alimentações e por onde consegue proteção territorial. O reconhecimento por parte da família a uma liderança religiosa, que acompanha os pais idosos de um usuário para visitá-lo em acolhimento noturno em um CAPS III. O significado para mulheres negras, atravessadas pelo sofrimento relacionado ao racismo, de encontros em espaços promovidos pelos movimentos sociais, como coletivos e rodas de conversas, onde podem falar de questões que sempre foram silenciadas. Assim como, quando um jovem deprimido que mal consegue sair de casa, diz que o seu dia foi mais "bacana", pois esteve em uma atividade esportiva ou uma roda de samba organizada pelos moradores do bairro.

Trouxemos aqui, situações do cotidiano com as redes denominadas como "informais" e ilustramos a importância que elas podem ter na vida dos sujeitos, por mais que por vezes essas atividades não sejam reconhecidas ou invisibilizadas diante da ênfase nos procedi- 
mentos centrados do cuidado em saúde e/ou em prol de uma formalidade da rede.

Contudo, não podemos perder de vista, a importância da luta pela consolidação e qualificação da assistência pelas políticas sociais, como educação, assistência social, cultura e lazer, trabalho e renda, como Malvezzi e Nascimento (2020) chamam atenção:

O trabalho na lógica intersetorial pressupõe não apenas uma articulação entre os setores públicos e privados. A parceria com os sujeitos que estão nos territórios, individualmente ou representados por movimentos sociais, amplia o conceito de intersetorialidade e possibilita o encontro com outros saberes e outras maneiras de compreensão do cotidiano. Além disso, a participação da comunidade nesses espaços tem o potencial de contribuir para a autonomia individual e o fortalecimento das lutas sociais; e de reafirmar o caráter democrático de participação dos sujeitos e instituições (MALVEZZI; NASCIMENTO, 2020, p. 5).

E assim, concluímos que, as redes ditas "informais', e espaços nos territórios, podem ser instrumentos para fortalecer o protagonismo e participação da população, inclusive elucidando o debate sobre a luta por políticas sociais e garantias dos direitos. Desse modo, a articulação dos serviços formais da rede com os pontos informais precisa ser desenvolvida de forma sistemática, devido à sua importância enquanto estratégia de cuidado, socialização e fortalecimento das redes sociais. Assim, é preciso reconhecer, apoiar e utilizar os pontos informais como componentes da rede a fim de fortalecer a autonomia e participação social dos usuários para a criação e manutenção desses espaços.

\section{O processo de trabalho do CAPS no período do COVID-19.}

Para falarmos do fortalecimento dessas redes formais e informais, do trabalho do CAPS no contexto atual, precisamos apontar que a pandemia, a despeito da sua gravidade, revela as contradições estruturais da sociedade capitalista e acirra a desigualdade entre os mais ricos e os pobres e miseráveis (BARBOSA; SILVA, 2020). 
Frente aos elementos expostos, que articulam dimensões estruturais da vida social e a conjuntura de crise política, econômica e pandêmica, já não há mais dúvidas de que a pandemia gera mudanças estruturais socioeconômicas e que implicam diretamente na vida dos sujeitos em seus cotidianos e nas possibilidades diárias frente a uma restrição de circulação no território e no convívio social.

Diante desse cenário, nos deparamos com as implicações e o que as construções teóricas e normativas ofertam de direção acerca do trabalho dos CAPS. Costa-Rosa (2000) nos sinaliza que o modelo da Atenção Psicossocial, por meio dos CAPS tem como desafio operacionalizar a desospitalização, desmedicalização, implicação subjetiva e sociocultural, participação, autogestão, interdisciplinaridade, interlocução, livre trânsito do usuário e da população e territorialização com integralidade, tendo como prática de trabalho uma direção oposta às características do modo asilar.

Contudo, apesar do potencial do CAPS para promover a desinstitucionalização, é necessário reconhecer que esse dispositivo não deve sozinho, tentar dar conta da complexidade que envolve as questões da saúde mental e a diversidade de situações inerentes à experiência da loucura e do sujeito na sua totalidade.

Sendo assim, para objetivar e contemplar estes princípios, neste momento de pandemia foi necessário repensar a reorganização do processo de trabalho e a reconfiguração dos fluxos de informações nos CAPS, uma vez que nosso trabalho contempla o território e a circulação pela cidade primando pelo desenvolvimento de práticas que acolham, vinculem e que, na medida do possível, possam propiciar a constituição de novos formatos de produção de ações individuais e coletivas.

Diante do aparecimento dos primeiros casos no Brasil de infecção por SARS-CoV-2, o MS deliberou novas recomendações na direção do isolamento e distanciamento social. Também destacamos que às orientações da Superintendência de Saúde Mental (SSM) do Rio de Janeiro, determinou em documento oficial, publicado em 16 de março, que às organizações da estrutura e rotinas internas dos serviços deveriam, além de atentar para a higienização dos CAPS e seus mobiliários, abordar ações educativas em saúde, voltadas à instrução da comunidade. 
Vale destacar, que no que tange o trabalho desenvolvido nos CAPS, e seguindo as indicações de evitar aglomerações para preservar os usuários e equipe, as ferramentas de trabalho - convivência, oficinas terapêuticas e atendimentos coletivos - foram suspensas, o que leva a perdas irreparáveis no que tange o cotidiano do trabalho e a potência dos espaços coletivos quanto produtores de cuidado. Bem como, ocorreram também mudanças nos demais serviços das diferentes políticas sociais.

E assim, emerge em nós a necessidade de manter e buscar estratégias mais radicais no âmbito de corresponsabilizar os acompanhamentos com os demais serviços do território, apostando em um cuidado compartilhado importante para assegurar a manutenção e estratégias de cuidado, e que vá na direção de apoiá-los visando enriquecer e expandir o trabalho na medida em que o território desenvolve um papel ativo na promoção da saúde mental.

Destacamos que o CAPS não preconiza o acompanhamento medicamentoso como única forma de tratamento, logo, deslocando a centralidade desta modalidade para àquelas relacionadas à atenção psicossocial. Porém, no contexto da pandemia, e os PTS de usuários que incluem a medicação assistida nos CAPS, foi imprescindível pensar junto com as redes formais e informais de apoio como seria feito a administração dessas medicações, uma vez que num primeiro momento havia uma direção clara quanto a necessidade do isolamento social.

Diminuiu-se a circulação dos usuários no território e no serviço, o que possibilitou a redução da exposição dos usuários a possíveis contaminações, proporcionando a otimização da força de trabalho uma vez que, com um menor quantitativo de frequentadores foi possível atender com mais cuidado os usuários que se apresentavam em crise e que demandavam atendimentos presenciais (BARBOSA; NASCIMENTO, et al., 2020).

Ao falarmos acerca do cuidado em saúde mental pensamos diretamente nas singularidades apresentadas na assistência e manejos dos casos, mas isso não deve excluir o que há de comum entre esses indivíduos para além do sofrimento psíquico, as múltiplas expressões da questão social que os tangenciam e os formam quanto sujeitos pertencentes de uma sociedade desigual. 
Dialogando com o cenário atual, estamos falando de indivíduos que dentro de suas especificidades possuem uma vulnerabilidade maior para a contaminação e agravamento da COVID-19, relacionada à condição psíquica que acaba por produzir desorganizações nas atividades diárias, maior exposição à situações de risco, dificuldades no autocuidado, alta taxa de comorbidades físicas em consequência do uso prolongado de medicação psiquiátrica, a negligência em relação ao adoecimento físico decorrente de longos períodos de internação em hospitais psiquiátricos e as barreiras de acesso às demais especialidades clínicas para além da saúde mental (BARBOSA; NASCIMENTO, et al., 2020). Que estão entrelaçadas com as condições de vida que incluem a ausência de água potável, saneamento básico, produtos de higiene, reflexos da falta de trabalho e renda, este último afetando diretamente nas condições de alimentação e moradia.

Nesse sentido, as apostas na intensividade do trabalho intersetorial em uma pandemia, provocadas pela radicalidade do cuidado territorial, apontou para as articulações com serviços da saúde, como a Clínica da Família e demais CAPS, assim como, o trabalho na lógica do acesso quanto sujeito de direitos. Nessa direção, foi primordial o resgate da cidadania por meio dos cuidados em saúde, mas para além disso, a articulação com outras instituições e serviços da rede intersetorial, como exemplo: o Centro de Referência da Assistência Social (CRAS), Centro de Referência Especializado da Assistência Social (CREAS), Organizações não Governamentais (ONG), e com atores do território, como familiares, amigos, vizinhos como rede de suporte e apoio.

Desta forma, cuidar na perspectiva da atenção psicossocial, rompendo com a lógica manicomial, exige experimentações de novos modos de cuidar do sujeito que precisa ir mais à frente das redes institucionais construídas e apostar também nas redes vivas que são produzidas no trabalho em acontecimento.

Nesse caminho aparece a importância do conhecimento territorial em suas múltiplas dimensões e a construção de novos territórios que deem sentido para o sujeito, o que possibilita a produção de cuidado, por meio do processo de construção de identidades, de vínculos, de laços sociais e de pertencimento dos espaços da cidade. 
Destacamos também que em alguns casos foram necessárias visitas domiciliares e institucionais como estratégias necessárias de atenção à crise, apesar de não preconizadas no período de pandemia pelo Ministério de Saúde. Ainda assim, a nossa presença no território é convocada diante da complexidade das situações vivenciadas pelos usuários da saúde mental que foram acentuadas frente ao isolamento social reforçando a necessidade de atendimento conjunto com os demais dispositivos.

Tudo isso nos possibilitou aquecermos redes de suporte no território fundamentando as ações no cuidado extramuros que produz redes de sociabilidade, para além do que é instituído pelos dispositivos, construindo diversas conexões na vida a partir dos encontros.

Logo, ao articularmos cotidianamente com as demais políticas setoriais, compreendendo o sujeito na sua totalidade e complexidade, reconhecemos o território como espaço de desenvolvimento de novas interações e de práticas de cuidado em rede.

\section{Considerações finais}

Ao longo deste artigo sustentamos que para que o trabalho aconteça nos CAPS é fundamental pensar a relação dos sujeitos com os territórios e consequentemente a intersetorialidade, a partir da incorporação de dispositivos que utilizados no cotidiano e produzidos pelos serviços, oportunizam a ressignificação dos espaços, construção de laços sociais, convívio social e abertura de espaços de diálogo e de produção de processos de trabalho em rede.

As mudanças necessárias diante da pandemia do COVID-19, nos trazem dois alertas, o trabalho intersetorial em "redes" compõe os processos multidisciplinares e interdisciplinares desenvolvidos nos CAPS mas devem estar para além destes, e a importância de não cairmos no equívoco de reduzir a intersetorialidade na atenção psicossocial à rede RAPS.

Reconhecer as redes construídas pelos sujeitos envolvidos no processo de cuidado e ter atenção a lógica e atribuições das diferentes políticas sociais, são fundamentais para reinventar as estratégias 
em prol da continuidade da radicalização do cuidado territorial na produção de saúde.

É importante destacar, que o trabalho intersetorial no campo atenção psicossocial, deve sim respeitar as particularidades dos usuários atendidos, dos territórios, dos serviços e profissionais, mas não pode ser considerado descolado do contexto político, econômico e social. Logo, defendemos que contribuir para fortalecimento das "redes", possibilita uma visão crítica da sociedade sendo este um instrumento político e social frente a luta por melhores condições de vida, uma vez que: "juntas, somos mais forte"!

\section{Referências}

AMARANTE, P. Novos sujeitos, novos direitos: o debate sobre a reforma psiquiátrica no Brasil. Cadernos de Saúde Pública, Rio de Janeiro, 11 (3): 491-494, jul/set, 1995.

. Saúde mental e atenção psicossocial. Rio de janeiro: Editora Fiocruz, 2007.

; NUNES, M. O. A reforma psiquiátrica no SUS e a luta por uma sociedade sem manicômios. Ciência \& Saúde Coletiva, v. 23, p.2067-2074, 2018.

BASAGLIA, F. A destruição do hospital psiquiátrico como lugar de institucionalização. In: AMARANTE, P. (Org). Franco Basaglia: escritos selecionados em saúde mental e reforma psiquiátrica. Rio de Janeiro: Garamond, 2005.

BARBOSA, A. S.; SILVA, A. P. O cuidado em saúde mental no cenário de pandemia da COVID-19: a experiência de (re)organização do CAPS UERJ. In: LOLE, A.; STAMPA, I.; GOMES, R. (Org.). Para além da quarentena: reflexões sobre crise e pandemia. Rio de Janeiro: Morula, 2020. Disponível em: <https://books.google.com.br/books?hl=ptBR\&I $r=\& i d=b 0 T q D w A A Q B A J \& o i=f n d \& p g=P T 249 \& d q=u e r j+a n d+>$. Acesso em: 03 set. 2020.

BARBOSA, A. S.; NASCIMENTO, C. V.; DIAS, L. B. S.; SANTO, T. B. E; CHAVES, R. C. S.; FERNANDES, T. C. Processo de trabalho e cuidado 
em saúde mental no Centro de Atenção Psicossocial da UERJ na pandemia de COVID-19. Brazilian Journal of Health Bioomedical Sciences - BJHBS, Rio de Janeiro, v. 19, n. 1, p. 11-19, 2020. Disponível em:<http://revista.hupe.uerj.br/WebRoot/pdf/711_pt.pdf $>$. Acesso em: 03 set. 2020.

BIDARRA, Z. S. Pactuar a intersetorialidade e tramar as redes para consolidar o sistema de garantia dos direitos. Serviço Social \& Sociedade, São Paulo, n. 99, p. 483-497, jul./set., 2009.

BRASIL. Ministério da Saúde. Portaria GM/MS n. 3.088, de 23 de dezembro de 2011. Institui a Rede de Atenção Psicossocial para pessoas com sofrimento ou transtorno mental e com necessidades decorrentes do uso de crack, álcool e outras drogas, no âmbito do Sistema único de Saúde, Brasília. Ministério da Saúde, 2011.

. Presidência da República. Decreto no 7.179, de 20 de maio de 2010. Institui o Plano Integrado de Enfrentamento ao Crack e outras Drogas, Cria o seu Comitê Gestor, e dá outras providências. Disponível em: <https://legislacao.presidencia.gov.br/atos/? tipo $=$ DEC \& numero $=7179 \&$ ano $=2010 \&$ ato $=602$ ETRU5EMVpWT8d6 $>$. Acesso em: 05 set. 2020.

. Ministério da Saúde. Portaria GM/MS no 3.588, de 21 de dezembro de 2017. Altera as Portarias de Consolidação no 3/GM/MS e no 6/GM/MS, de 28 de setembro de 2017, para dispor sobre a Rede de Atenção Psicossocial, dá outras providências e inclui procedimentos de Tratamento em Psiquiatria na Tabela de Procedimentos, Medicamentos, Órteses, Próteses e Materiais Especiais do SUS. Disponível em: <https://bvsms.saude.gov.br/bvs/saudelegis/gm/2017/ prt3588_22_12_2017.html>. Acesso em: 05 set. 2020.

. Ministério da Saúde. Saúde mental no SUS: os centros de atenção psicossocial. Brasília: Ministério da Saúde, 2004.

COE, N. M.; DUARTE, M. J. de O. A construção da atenção psicossocial na pública de Saúde Mental no Brasil: Rupturas, Tessituras e Capturas. In: DUARTE, M. J. de O.; PASSOS, R. G.; GOMES, T. M. da S. (Org.). Serviço Social, saúde mental e drogas. Campinas: Papel Social, 2017. 
COSTA-ROSA, A. O modo psicossocial: um paradigma das práticas substitutivas ao modo asilar. In: AMARANTE, P. (Org.). Ensaios: subjetividade, saúde mental, sociedade. Rio de Janeiro: Editora Fiocruz. 2000.

DUARTE, M. J. de O. O campo psicossocial na política de saúde mental brasileira: uma análise micropolítica da produção do cuidado em saúde mental. In: Anais do XI Congresso Luso-Afro-Brasileiro de Ciências Sociais. 07 a 10 de agosto, Salvador: UFBA, 2011. Disponível em:< http://www.xiconlab.eventos.dype.com.br>. Acesso em: 06 set. 2020.

. Diversidade sexual e de gênero e saúde mental: enlaçando políticas e direitos. In: PASSOS, R. G.; COSTA, R.; SILVA, F. (Org.) Saúde mental e os desafios atuais da atenção psicossocial. Rio de Janeiro: Gramma, 2017.

. Política de saúde mental e drogas: desafios ao trabalho profissional em tempos de resistência. Libertas, Juiz de Fora, v. 18, n. 2, p. 277-243, ago./dez., 2018. Disponível em:_<https://periodicos.ufjf.br/index.php/libertas/article/view/18604>. Acesso em: 05 set. 2020. GUIMARÃES, T. A. A.; ROSA, L. C. S. A remanicomialização do cuidado em saúde mental no Brasil no período de 2010-2019: análise de uma conjuntura antirreformista. O Social em Questão, Ano XXII, n. 44, p. 111-138, mai./ago., 2019. Disponível em: <http://osocialemquestao.ser.puc-rio.br/media/OSQ_44_art5.pdf>. Acesso em 05 set. 2020.

NICÁCIO, F.; CAMPOS, G. W. S. Instituições de "portas abertas": novas relações usuários-equipes-contextos na atenção em saúde mental de base comunitária/territorial. Revista Terapia Ocupacional da USP, São Paulo, v. 16, n. 1, p. 40-46, 2005. LANCETTI, A. Clínica peripatética. 4. ed. São Paulo: HUCITEC, 2009.

PASSOS, R. G. Luta antimanicomial no cenário contemporâneo: desafios atuais frente à reação conservadora. Sociedade em Debate, Pelotas, v. 23, n. 2, p. 55 - 75, jul./dez, 2017.

. "Holocausto ou navio negreiro?": inquietações para a reforma psiquiátrica brasileira. Argumentum, Vitória, n. 10, v. 3, p. 10-22, set./dez. 2018. 
PEREIRA, M. de 0.; PASSOS, R. G. Luta antimanicomial e feminismos: discussões de gênero, raça e classe para a reforma psiquiátrica brasileira. Rio de Janeiro: Editora Autografia, 2017.

PEREIRA, S. L. B. Saúde mental e intersetorialidade: Reflexões a partir de demandas aos assistentes sociais. Ser Social, v.22, n.46, p.72-98, 27 jan., 2020.

PREFEITURA DO MUNICÍPIO DO RIO DE JANEIRO. Secretaria Municipal de Saúde. Resolução n. 4333, de 8 de março de 2020. Recomendações para os CAPS do município do Rio de Janeiro referentes a COVID-19. Diário Oficial [do] Município do Rio de Janeiro, 18 março, 2020, p.10-11.

ROSA, L. C. dos S.; LUSTOSA, A. Afinal, o que faz o Serviço Social na saúde mental? Serviço Social \& Saúde, Campinas, v. 11, n. 1 (13), p. 27-50, jan./jun., 2012. Disponível em:<https://periodicos.sbu.unicamp.br/ojs/index.php/sss/article/view/8635023>. Acesso em: 06 set. 2020.

ROTELLI, F. Formação e construção de novas instituições em saúde mental. In: AMARANTE, P.; CRUZ, L. B. (Org.). Saúde mental: formação e crítica. Rio de Janeiro: Ed. Fiocruz, 2008.

SCHEFFER, G.; SILVA, L. G. Saúde mental, intersetorialidade e questão social: um estudo na ótica dos sujeitos. Serviço Social \& Sociedade, São Paulo, n. 118, p. 366-393, jun.,2014.

SILVA, S. F. da; MAGALHÃES JÚNIOR, H, M. M. Redes de atenção à saúde: importância e conceitos. IN: SILVA, S. F. da et. al. (Org.). Redes de atenção à saúde no SUS: o pacto pela saúde e redes regionalizadas de ações e serviços de saúde. São Paulo: IDISA; CONASEMS, 2008.

YASUI, S. Rupturas e encontros: desafios da reforma psiquiátrica brasileira. Rio de Janeiro: Editora Fiocruz, 2010.

WANDERLEY, M. B; MARTINELLI, M. L.; PAZ, R. D. O. Intersetorialidade nas políticas públicas. Serviço Social \& Sociedade, São Paulo, n.137, p.7-13, jan./abr., 2020. 